\title{
Aceptabilidad Sensorial de Productos de Panadería y Repostería con Incorporación de Frutas y Hortalizas Deshidratadas como Ingredientes Funcionales
}

\section{Hilda H. Estrada-López ${ }^{(1) \star}$, Claudia E. Restrepo- Flórez $^{(2)}$ y María A. Iglesias-Navas ${ }^{(3)}$}

(1) Univ. Simón Bolívar de Barranquilla, Facultad de Administración y Negocios. Carrera 65 No. 64-17

Barranquilla- Colombia. (e-mail: hileslo@hotmail.com)

(2) Instituto de Ciencia y Tecnología Alimentaria - INTAL, Itagüí - Colombia. Carrera 50 G Nro. 12 sur -91 Itagüí - Colombia. (e-mail: crestrepo@intal.org)

(3) Universidad Simón Bolívar de Barranquilla, Facultad de Ingenierías, Barranquilla, Colombia.

(e-mail: miglesias1@unisimonbolivar.edu.co)

* Autor a quien debe ser dirigida la correspondencia

Recibido Nov. 15, 2017; Aceptado Ene. 16, 2018; Versión final Feb. 20, 2018, Publicado Ago. 2018

\section{Resumen}

Se presentan resultados de estudio sobre preferencias de consumo de productos de panadería y repostería en diferentes localidades de la ciudad de Barranquilla. Se desarrollaron formulaciones de los productos de mayor preferencia. Estos son pan molde $(P)$ y ponqué $(P Q)$ adicionados con mango, guayaba y/o ahuyama en polvo en sustitución parcial de la harina de trigo, como fuente natural de sabor, vitaminas y fibra dietaria. Los prototipos de $\mathrm{P}$ y $\mathrm{PQ}$ se sometieron a pruebas de calidad microbiológica, fisicoquímica y sensorial a través de una prueba afectiva de nivel de agrado, usando escala hedónica de 5 puntos. La mayor aceptabilidad sensorial se logró para el pan tajado con ahuyama-mango y el ponqué con ahuyama, basados principalmente en los atributos de apariencia y sabor $(p<0.05)$. Así mismo, los prototipos obtenidos presentaron un aporte importante de fibra dietaria, con $20 \%$ de ingesta adecuada para el pan y el $11 \%$ para el ponqué.

\section{Sensorial Acceptability of Bakery and Pastry Products with Incorporation of Dehydrated Fruits and Vegetables as Functional Ingredients}

\begin{abstract}
A study on consumption preferences of bakery products produced in different locations in Barranquilla Colombia. Formulations of the most preferred products were done: sandwich-bread (P) and butter cake (PQ) added with mango, guava and pumpkin powder in partial replacement of wheat flour, as a natural source of flavor, vitamins and dietary fiber. The prototypes of $P$ and $P Q$ were subjected to microbiological, physicochemical and sensorial affective test, using a 5-point hedonic scale. The highest sensory acceptability was achieved for bread with pumpkin-mango and cake with pumpkin, based mainly on appearance and taste attributes $(p<0.05)$. Likewise, the prototypes presented an important contribution of dietary fiber, with $20 \%$ of adequate intake for the bread and $11 \%$ for the cake.
\end{abstract}




\section{INTRODUCCIÓN}

En los últimos años, las tendencias mundiales de la alimentación muestran un gran interés hacia productos alimenticios que aporten un alto valor nutricional y generen beneficios en la salud humana (Beal et al., 2017; Bigliardi y Galati, 2013; Santeramo et al., 2017). La fibra dietaria como componente alimentario, ha permitido el desarrollo de una gran variedad de productos e ingredientes funcionales, debido a que juegan un papel importante en la prevención y tratamiento de enfermedades tales como cáncer colorrectal, obesidad, ateroesclerosis, diabetes, entre otros (Vergara et al., 2007). Las frutas son fuente potencial de fibra y antioxidantes, también aportan minerales y vitaminas necesarios en la dieta (Vergara et al., 2007). El consumo elevado de frutas tiene un impacto positivo en la salud, debido a la presencia de metabolitos capaces de neutralizar especies reactivas del oxígeno (Prabu et al., 2012), sin embargo, en Colombia, el consumo de frutas y vegetales ha ido disminuyendo en los últimos años, donde el $71,9 \%$ de los habitantes no consumen frutas ni verduras diariamente. Según Vera y Hernández (2013), manifiestan que en la última Encuesta Nacional de Situación Nutricional en Colombia ENSIN 2010, uno de cada tres colombianos, entre los 5 y los 64 años, no consume frutas diariamente, y 5 de cada 7 no consumen hortalizas o verduras al día.

Los desperdicios de las frutas y verduras son abundantes debido a malas prácticas de manejo durante la producción agrícola, daños en los cultivos, cambios climáticos, aspectos de maduración, transporte y conservación como también aspectos sensoriales ligados a cambios en las características organolépticas de los alimentos y la vida útil, o cambios de preferencias de los consumidores (Martínez y Quintero, 2017). Debido a esto, los métodos de secado se han convertido en una alternativa de conservación y aprovechamiento de los nutrientes presentes en las frutas y vegetales que permitan minimizar la cantidad de residuos y desperdicios durante la cadena productiva y permita el crecimiento y desarrollo de una amplia variedad de productos funcionales y de alta calidad (O'Shea et al., 2012; Martins et al., 2017). El mango (Manguifera indica L.) es una fruta conocida y de alto consumo, que contiene calcio, fósforo, hierro, sodio, potasio, vitamina A, tiamina, riboflavina, niacina y ácido ascórbico (Ribeiro et al, 2007).

Por su parte la guayaba (Psidium guajava) tiene un alto contenido de polifenoles y su consumo frecuente permite mantener un adecuado equilibrio oxidativo, también es una buena fuente de vitamina $C$, vitamina $A$, fósforo, hierro y calcio. A su vez, la guayaba también ha sido considerada como una buena fuente de fibra dietaria (Zapata et al., 2013; Hidalgo et al., 2015; Jimenez et al., 2001). La ahuyama es rica en carotenos, pectina, minerales y otras sustancias de beneficio para la salud (Morris et al., 1998). El uso intensivo de la harina de trigo blanca, en productos de panadería y repostería, ha conllevado a menores aportes de fibra dietaria y reconocida deficiencia de aminoácidos como la lisina y la treonina en comparación con otras fuentes alimentarias como la leche, los guisantes, la soya, entre otros (Ndife et al., 2011). En respuesta a esto, diversas industrias de alimentos buscan satisfacer el mercado con productos funcionales y productos innovadores con formas, colores y texturas novedosas, basándose en las necesidades actuales y exigencias de los consumidores (Palacio et al., 2017). Se ha encontrado que los productos de panadería proporcionan una matriz ideal para la adición de ingredientes funcionales debido a que no presenta mayores implicaciones tecnológicas en su elaboración y pueden incrementar su valor nutricional y funcional (Morris et al., 1998).

A nivel sensorial, atributos como apariencia, color, olor, sabor, textura en todos los productos, juegan un papel importante que definen la calidad y aceptabilidad de los mismos, así como la acción de re-compra (Grillo et al., 2014). Por lo anterior, se hace necesario realizar estudios de aceptabilidad sensorial donde se pretende determinar diferencias entre los productos en cuanto a la aceptación del consumidor. Comúnmente, se utilizan escalas hedónicas de 9 puntos, y sus variantes de 7, 5 y 3 puntos (Ramírez, 2012). El número de consumidores recomendados por literatura para ejecutar en laboratorios es de 25-50 evaluadores, para ubicación central 100 o más respuestas por producto y 50-100 familias para prueba en el hogar (Stone y Sidel, 2004; Hough et al, 2006). El objetivo de esta investigación fue incorporar mango (Mangifera indica), guayaba (Psidium guajava) y ahuyama (Cucurbita moschata) deshidratadas como ingredientes funcionales en productos de panadería y repostería identificados en un estudio de preferencias de consumo, realizado en la ciudad de Barranquilla- Colombia, y evaluar su nivel de aceptabilidad sensorial con consumidores.

\section{MATERIALES Y MÉTODOS}

Esta investigación se realizó en dos etapas, la primera correspondiente a un estudio de mercado y la segunda a la evaluación de aceptabilidad sensorial de prototipos de productos de panadería y repostería con adición de mango, guayaba y/o ahuyama, procedente de pequeños productores agrícolas del departamento del Atlántico.

\section{Estudio de preferencia de consumo}

Se realizó una encuesta con enfoque descriptivo-cuantitativo, para identificar objetivamente las expectativas y preferencias del consumidor potencial. La población sujeta a estudio, comprendió personas mayores de 18 
años residentes en las diferentes localidades de la Ciudad de Barranquilla. El tamaño de la muestra calculada fue de 384 personas, con un nivel de confianza del 95\% y un error del 5\%. Se construyó un cuestionario sociodemográfico de los participantes y se aplicó una encuesta para dos líneas de productos (panadería y repostería), con el fin de evaluar las expectativas, motivación y hábitos de consumo.

\section{Materia prima}

Los materiales vegetales de mango (Mangifera indica), guayaba (Psidium guajava) y ahuyama (Cucurbita moschata), procedentes del municipio de Santa Lucía (Atlántico - Colombia), fueron sometidos a proceso de secado en un horno de convección forzada a $50^{\circ} \mathrm{C}$ por 12 horas hasta alcanzar un nivel máximo de $10 \%$ de humedad. La materia prima en polvo obtenida, se le determinó actividad de agua (aw) a $25^{\circ} \mathrm{C}$ empleando el método AOAC 978.18 y el porcentaje de humedad mediante Termobalanza (Ohaus MB-200) a $105^{\circ} \mathrm{C}$.

\section{Formulaciones de los prototipos}

Se obtuvieron prototipos a escala piloto en el INTAL de pan molde, comúnmente llamado "pan tajado" con adición de ahuyama y mango en polvo (P1), con ahuyama y guayaba en polvo (P2), y pan tajado con mango y guayaba en polvo (P3). Los prototipos de ponqué (variedad de cake, presentado en forma rectangular y tajado) con mango en polvo (PQ1), con ahuyama en polvo (PQ2) y ponqué con guayaba en polvo (PQ3).

\section{Formulación de pan y ponqué}

A una fórmula abierta de pan molde constituida por harina de trigo panadera $100 \%$, agua $50 \%$, margarina industrial $10 \%$, azúcar $10 \%$, sal 2,0\% y levadura instantánea 2,0\%; se le sustituyó un $10 \%$ de la harina con la mezcla ahuyama-mango en polvo en relación 7:3; del mismo modo para la mezcla ahuyama -guayaba. Para el caso de la mezcla mango- guayaba en polvo se sustituyó solo el 6\% en relación 3:3. A una fórmula abierta de ponqué constituida por harina de trigo repostera $100 \%$, margarina industrial $90 \%$, huevos frescos $80 \%$, azúcar $80 \%$, leche entera $55 \%$, polvo de hornear $2 \%$, esencia de vainilla $0,5 \%$ y sal $0,3 \%$; se le sustituyó a cada formulación de ponqué, un $20 \%$ de la harina de trigo por mango en polvo, ahuyama en polvo, guayaba en polvo, respectivamente. Los porcentajes de sustitución fueron determinados a través de pre- ensayos.

Para ambos productos se aplicó un proceso de elaboración estandarizado, en caso del pan (amasado de todo los ingredientes en amasadora de dos tiempos por 6 minutos usando agua a $10^{\circ} \mathrm{C}$, reposo por 15 minutos a $23^{\circ} \mathrm{C}$, porcionado por $600 \mathrm{~g}$, fermentación en molde por 90 minutos a $30^{\circ} \mathrm{C} / 75 \% \mathrm{HR}$, horneado por convección forzada a $170^{\circ} \mathrm{C}$ por 30 minutos en horno rotatorio) y para el ponqué (cremado de la margarina con el azúcar por 4 minutos a velocidad alta, incorporación de los huevos y del resto de ingredientes secos, batido por 4 minutos a velocidad baja, adición de la leche y batido final por 1 minutos a velocidad media, servido en moldes por $500 \mathrm{~g}$, horneado por conducción a $170^{\circ} \mathrm{C}$ por 35 minutos en horno gavetero, enfriamiento, desmoldado porcionado), para ambos productos se procedió a empacar individualmente usando una película de Polipropileno Biorientado (BOPP) de 40 micras sellable, se enfriaron rápidamente en abatidor hasta $4^{\circ} \mathrm{C}$, para ser remitidos en contenedores isotérmicos hacia la ciudad de Barranquilla.

\section{Evaluación de calidad de los prototipos}

Los productos fueron analizados fisicoquímica y microbiológicamente, al siguiente día de su elaboración. La actividad de agua $\left(a_{w}\right)$ y la humedad fue medida como se mencionó anteriormente. Los parámetros microbiológicos realizados fueron: recuento de Bacillus cereus (ISO 7932), recuento de Staphylococcus aureus (ISO 6881-1), Número Más Probable (NMP) de coliformes totales y fecales (ISO 4831), recuento de mohos y levaduras (ISO 21527-1) y Detección Salmonella spp (ISO 6579).

\section{Prueba sensorial afectiva de nivel de agrado}

Los prototipos se conservaron en refrigeración en cuanto se obtuvieron los resultados de las pruebas microbiológicas (5 días después de la elaboración). La prueba se realizó con 45 consumidores potenciales de diferentes localidades de la ciudad de Barranquilla, y se ejecutó en un laboratorio de la Universidad Simón Bolívar de la misma ciudad. La población objeto de estudio para la prueba del nivel de agrado de los prototipos de $P$ y $P Q$, comprendió edades de 15 a 20 años en un 7,81\%, de 21 a 30 años en 18,75\%, de 31 a 40 años en $34,38 \%$ y personas mayores de 40 años en $39,06 \%$. El 65,6 \% de los participantes fueron mujeres y el $34,4 \%$ hombres.

Se realizó una prueba afectiva empleando una escala hedónica verbal de cinco puntos correspondientes a distintos grados de satisfacción, equilibrados alrededor de un punto neutro, donde se le pide al consumidor marcar la respuesta que mejor refleje su opinión acerca de la apariencia, sabor y textura. A los participantes 
se les entregaron dos series de tres muestras, la primera, correspondiente a los prototipos de pan P1 - P2- P3 y la segunda, a los prototipos de ponqué PQ1- PQ2- PQ3. Las muestras fueron identificadas con códigos de tres dígitos aleatorios.

\section{Análisis compuestos de interés nutricional}

A los materiales en polvo y los prototipos de mayor nivel de agrado, se les determinó el contenido de fibra dietaria (\%FD) empleando el método enzimático AOAC 985.29. El contenido de Calcio (Ca) y Hierro ( $\mathrm{Fe})$ de acuerdo al método AOAC 999.10. La determinación de vitamina C (Vit C) y vitamina A (Vit A), se realizó de acuerdo al protocolo establecido por Moron et al., 1997. Para cálculos de aportes nutricionales se utilizaron los valores de ingesta adecuada (IA) para fibra de $25 \mathrm{~g} /$ día. Aporte Dietético Recomendado (RDA) de Vitamina C de 60 mg/día, vitamina A de 2333 IU o 700 g/día, calcio 1000 mg/día y para hierro 18 mg/día.

\section{Análisis Estadístico}

El análisis de los datos de preferencia de consumo se realizó mediante el uso del software estadístico SPSS v. 19. Los datos de la prueba sensorial de nivel de agrado, se analizaron usando un software estadístico Statgraphics Centurion XV, realizando una prueba de Friedman para establecer diferencias estadísticamente significativas entre los prototipos con base en la asignación de un valor numérico a escala verbal: me gusta mucho (5), me gusta (4), ni me gusta ni me disgusta (3), me disgusta (2), me disgusta mucho (1), y la prueba de Wilcoxon para comparaciones pareadas entre los prototipos por atributo (Ramírez, 2012; Stone y Sidel, 1992).

\section{RESULTADOS Y DISCUSIÓN}

En relación a los resultados del estudio de preferencias de consumo, los participantes correspondieron a $59,5 \%$ de mujeres y el $40,5 \%$ hombres. La actividad principal de los encuestados muestra un $26,2 \%$ empleados, $22,2 \%$ oficios del hogar y el $19,2 \%$ estudiantes. Sobre el estado civil, el $50,5 \%$ eran casados o en unión libre y $37,8 \%$ solteros. Con respecto a los hábitos de compra, la línea de panadería fue la de mayor compra o consumo con un $72 \%$, en comparación con la línea de repostería con $27,3 \%$. En cuanto a la motivación de compra, los encuestados atribuyeron mayor importancia a los factores sabor $(85,4 \%)$, la marca (76\%), el precio (72\%); y menos importancia son el envase (53,9\%), el nombre $(46,6 \%)$ y la publicidad $(45 \%)$. Los resultados en la línea de panadería mostraron una alta preferencia en productos como pan tajado (44,5\%), seguido de panecillo $(34,2 \%)$ y por último la mogolla $(21,3 \%)$. En la línea de repostería, los participantes mostraron mayor preferencia por galleta tipo cracker salada $(27,9 \%)$, seguido de ponqué $(23,6 \%)$. Para ambas líneas de productos, se prefirió la harina tradicional $(65 \%)$ frente a la harina integral; endulzante tradicional $(52,5 \%)$ frente a la miel y los edulcorantes no calóricos, y los sabores a guayaba $(42 \%)$ y mango $(20,2 \%)$ con respecto a limón, picante, ají y cebollín.

En la línea de repostería se optó por desarrollar ponqué (segundo de mayor preferencia) a razón de las implicaciones tecnológicas para el escalamiento del proceso de galleta tipo cracker salada.

\section{Materiales vegetales en polvo y formulaciones de los prototipos}

Los compuestos de interés nutricional y parámetros fisicoquímicos para los materiales vegetales en polvo obtenidos por deshidratación con aire caliente se presentan en la tabla 1. Se destacan los contenidos de fibra Dietaria para guayaba en polvo y de vitamina $C$ en el mango, así mismo los valores de vitamina $A$ en la ahuyama resultan superiores a los aportados por las frutas utilizadas en esta investigación. De estos resultados sobresale los valores de FD, los autores Morris et al. (1998) encontraron en la ahuyama en polvo, la pectina como constituyente mayoritaria de la fibra soluble y en la fracción insoluble la celulosa, atribuyéndole un mejoramiento en las características de textura en el pan cuando se incorporó aún en bajos niveles.

El valor de FD fue superior al reportado por Guiné et al. (2011) y por Saeleaw y Schleining (2011), para la ahuyama en polvo obtenida en condiciones similares a esta experimentación, en contraste con solo una tercera parte de la vitamina A reportada por los últimos. La actividad de agua es similar a la obtenida por Keen et al. (2016) de 0,333 . El valor de calcio encontrado en $100 \mathrm{~g}$ de ahuyama en polvo representa el $18 \%$ del RDA (calcio $1000 \mathrm{mg} / \mathrm{día}$ ).

Los parámetros fisicoquímicos de los prototipos obtenidos se muestran en la tabla 2. El rango de humedad en el pan tajado estuvo entre 25- 28\%, inferior al indicado por See et al. (2007), para una sustitución igual al experimentado del $10 \%$ en harina de ahuyama (34,25\%), ésto se atribuye a una alta capacidad de retención de agua según Saeleaw y Schleining (2011). Los valores de actividad de agua obtenidos para el ponqué son similares 0,828 , pero son inferiores para el pan $(0,950)$ reportados por Pongjanta et al. $(2006)$. 
Tabla 1: Compuesto de interés nutricional de los materiales en polvo

\begin{tabular}{lccccccc}
\hline \multicolumn{1}{c}{ Producto } & $\% H$ & aw & $\% F D$ & $\begin{array}{c}C a \\
(m g / 100 g)\end{array}$ & $\begin{array}{c}F e \\
(m g / 100 g)\end{array}$ & $\begin{array}{c}\text { Vit C } \\
(\mathrm{mg} / 100 \mathrm{~g})\end{array}$ & $\begin{array}{c}\text { Vit A } \\
(\mathrm{Ul} / 100 \mathrm{~g})\end{array}$ \\
\hline Ahuyama en polvo & 5,21 & 0,362 & 15,87 & 184,9 & 1,56 & $<1,87$ & 24,8 \\
Guayaba en polvo & 6,44 & 0,345 & 44,44 & 65,56 & 0,93 & 32,14 & 3,6 \\
Mango en polvo & 5,85 & 0,287 & 10,98 & 46,17 & 4,42 & 87,21 & 2,1 \\
\hline
\end{tabular}

Tabla 2: Análisis fisicoquímico de los prototipos

\begin{tabular}{lcc}
\hline \multicolumn{1}{c}{ Producto } & $\% \mathrm{H}$ & $\mathrm{a}_{w}$ \\
\hline P1 Pan tajado Ahuyama - mango & 27,85 & 0,902 \\
P2 Pan tajado Ahuyama - guayaba & 25,06 & 0,878 \\
P3 Pan tajado Mango - guayaba & 26,61 & 0,894 \\
PQ1 Ponqué Mango & 24,62 & 0,882 \\
PQ2 Ponqué Ahuyama & 22,70 & 0,850 \\
PQ3 Ponqué Guayaba & 25,30 & 0,870 \\
\hline
\end{tabular}

Los recuentos microbiológicos de los prototipos se encontraron cumpliendo los requisitos establecidos en la legislación Colombiana para Recuento de Bacillus cereus $<100 \mathrm{UFC/g}$, Recuento de Staphylococcus aureus $<100$ UFC/g, NMP de coliformes totales y fecales $<3 \mathrm{NMP} / \mathrm{g}$, Recuento de mohos y levaduras $<200 \mathrm{UFC} / \mathrm{g}$, y Ausencia de Salmonella spp.

\section{Nivel de aceptación de los prototipos}

En la tabla 3, se presenta la moda y los estadísticos de la prueba de Friedman y Wilcoxon de los datos del nivel de agrado de los prototipos de pan tajado y ponqué. La apariencia y el sabor fueron estadísticamente diferentes para los prototipos de pan molde ( $p$ valor $<0,05)$, y sólo la apariencia para el caso del ponqué. Los valores de la moda indicaron calificaciones entre los niveles de escala de "me gusta mucho - 5" y "me gusta - 4", excepto para el sabor de P3 y apariencia del PQ3, la cual resultó ser indiferente ("Ni me gusta, ni me disgusta - 3"), para los evaluadores.

La prueba de Wilcoxon permitió determinar cuáles pares de prototipos son estadísticamente diferentes, siendo P1/P3 en apariencia y sabor; P2/P3, PQ1/PQ3, y PQ2/PQ3 en apariencia únicamente, esto se debe a la incorporación de la guayaba en polvo en los prototipos P3 y PQ3, la cual impartió color oscuro y sabor ácido para el caso de P3.

La buena aceptabilidad de los prototipos $\mathrm{P} 1$ y PQ2, se atribuye a la función resaltadora del color que le aportó la ahuyama en polvo, lo cual resalta la apariencia con respecto a los productos comerciales, y le confiere buenas características sensoriales de sabor y textura. Morris et al. (1998), demostraron el aumento del nivel de aceptabilidad sensorial de panes adicionados con ahuyama en polvo, fuertemente correlacionados con el aumento del volumen específico atribuido a la funcionalidad de la fibra en esa matriz. En contraste, Pongjanta et al. (2006), encontraron un efecto negativo sobre la aceptabilidad sensorial en formulaciones de pan tipo sandwich con más de $20 \%$ de pulpa de ahuyama, y del $30 \%$ en fórmulas de ponqué.

Los resultados estadísticos de la prueba sensorial, permitieron establecer la apariencia como el atributo de más influencia en la aceptabilidad, directamente relacionado con el color, y se explica dado que los consumidores prestan más atención a los aspectos llamativos con base en las características visuales y a partir de esto genera un criterio de calidad acerca del producto (Ramírez y Castro, 2014). 
Tabla 3: Prueba de Friedman y Wilcoxon para las características sensoriales de los prototipos de pan tajado y ponqué

\begin{tabular}{|c|c|c|c|c|c|c|c|c|c|c|}
\hline \multicolumn{6}{|c|}{ Pan molde } & \multicolumn{5}{|c|}{ Ponqué } \\
\hline \multirow{2}{*}{ Atributos } & \multicolumn{2}{|c|}{ Prueba Friedman } & \multirow{2}{*}{ Moda } & \multicolumn{2}{|c|}{ Prueba Wilcoxon } & \multicolumn{2}{|c|}{ Prueba Friedman } & \multirow{2}{*}{ Moda } & \multicolumn{2}{|c|}{ Prueba Wilcoxon } \\
\hline & Pan molde & $\begin{array}{l}\text { Rangos } \\
\text { promedios }\end{array}$ & & Muestras & P- valor & Ponqué & $\begin{array}{l}\text { Rangos } \\
\text { promedios }\end{array}$ & & Muestras & P- valor \\
\hline \multirow[t]{4}{*}{ Apariencia } & P1 & 2,18 & 5 & $\mathrm{P} 1 / \mathrm{P} 2$ & 0,233 & PQ1 & 2,12 & 5 & PQ1/PQ2 & 0,657 \\
\hline & $\mathrm{P} 2$ & 2,04 & 5 & P1/P3 & 0,001 & PQ2 & 2,20 & 5 & PQ1/PQ3 & 0,017 \\
\hline & P3 & 1,78 & 4 & P2/P3 & 0,045 & PQ3 & 1,68 & 3 & PQ2/PQ3 & 0,004 \\
\hline & $p$ - valor & 0,012 & - & - & - & $p$ - valor & 0,010 & - & - & - \\
\hline \multirow{4}{*}{ Sabor } & $\mathrm{P} 1$ & 2,24 & 5 & $\mathrm{P} 1 / \mathrm{P} 2$ & 0,142 & PQ1 & 1,89 & 4 & PQ1/PQ2 & 0,155 \\
\hline & P2 & 2,02 & 4 & P1/P3 & 0,003 & PQ2 & 2,26 & 4 & PQ1/PQ3 & 0,507 \\
\hline & P3 & 1,73 & 3 & P2/P3 & 0,170 & PQ3 & 1,85 & 5 & PQ2/PQ3 & 0,065 \\
\hline & $p$ - valor & 0,002 & - & - & - & $p$ - valor & 0,070 & - & - & - \\
\hline \multirow{4}{*}{ Textura } & P1 & 2,09 & 5 & $\mathrm{P} 1 / \mathrm{P} 2$ & 0,354 & PQ1 & 2,12 & 4 & PQ1/PQ2 & 0,702 \\
\hline & P2 & 1,93 & 4 & P1/P3 & 0,430 & PQ2 & 2,11 & 5 & PQ1/PQ3 & 0,109 \\
\hline & P3 & 1,98 & 5 & P2/P3 & 0,874 & PQ3 & 1,77 & 4 & PQ2/PQ3 & 0,227 \\
\hline & $p$ - valor & 0,492 & - & - & - & $p$ - valor & 0,054 & - & - & - \\
\hline
\end{tabular}

Compuestos de interés nutricional en los prototipos de mayor nivel de agrado

Los prototipos de mayor nivel de agrado presentaron contenidos de fibra dietaria, vitaminas y minerales indicados en la tabla 4, el aporte de fibra dietaria corresponde al $20 \%$ IA para el pan, y el $11 \%$ IA para el ponqué. See et al. (2007) y Păucean y Man (2014), reportaron un aumento significativo de la fibra cruda en formulaciones de pan incorporando ahuyama en polvo en niveles de 5, 10 y $15 \%$, y de pulpa de ahuyama en niveles de 15, 30 y $50 \%$, respectivamente, todos con respecto a una fórmula control. Los bajos valores obtenidos de vitamina $\mathrm{A}$ y $\mathrm{C}$ en los prototipos de pan y ponqué, obedecen a la dosis utilizada de las fuentes (frutas y hortalizas en polvo) y a su naturaleza lábil.

Tabla 4: Compuestos de interés nutricional de los prototipos de mayor nivel de agrado

\begin{tabular}{|l|c|c|}
\hline Nutriente & $\begin{array}{c}\text { Pan tajado Ahuyama - mango } \\
P 1\end{array}$ & $\begin{array}{c}\text { Ponqué Ahuyama } \\
\text { PQ2 }\end{array}$ \\
\hline Fibra dietaria \% & 5,04 & 2,65 \\
\hline Vitamina A Ul/100g & $<2,0$ & $<2,0$ \\
\hline Vitamina C mg/100g & $<1,87$ & $<1,87$ \\
\hline Calcio $\mathrm{mg} / 100 \mathrm{~g}$ & 50,06 & 59,29 \\
\hline Hierro $\mathrm{mg} / 100 \mathrm{~g}$ & 2,31 & 0,77 \\
\hline
\end{tabular}

\section{CONCLUSIONES}

Los niveles de incorporación del mango, guayaba y ahuyama en polvo en los productos de panadería y repostería, resultaron apropiados para los productos, obteniendo una aceptabilidad sensorial superior para el pan tajado con ahuyama-mango (P1) y el ponqué con ahuyama (PQ2), basados principalmente en los atributos de apariencia y sabor, contrario a los resultados obtenidos en el estudio de preferencias realizado inicialmente en esta investigación (preferencia de sabor a guayaba en 42\%), los prototipos P2, P3 y PQ3, 
incorporados con guayaba, no obtuvieron alta aceptación sensorial, poniendo a la ahuyama en un nivel promisorio para ser incorporada en gran variedad de productos, así mismo, los prototipos de mayor aceptación presentaron un aporte importante de fibra dietaria $20 \%$ IA para el pan, y el $11 \%$ IA para el ponqué, en contraste con la vitamina $\mathrm{A}$ y $\mathrm{C}$, los cuales se atribuyen a la cantidad utilizada de las fuentes (frutas y hortalizas en polvo) y a su naturaleza lábil.

\section{REFERENCIAS}

Association of Oficial Analytical Chemists (AOAC), Official Methods of Analysis, United States of America (1996)

Beal, T., E. Massiot, J.E. Arsenault, M.R. Smith, e R.J., Hijmans, Global Trends in Dietary Micronutrient Supplies and Estimated Prevalence of Inadequate Intakes, doi: 10.1371/journal.pone.0175554, PloS one, 12(4) (2017)

Bigliardi, B. y F. Galati, Innovation Trends in the Food Industry: The Case of Functional Foods, doi: 10.1016/j.tifs.2013.03.006, Trends in Food Science \& Technology, 31(2), 118-129 (2013)

Grillo, O., V. Rizzo, R. Saccone, B. Fallico, A. Mazzaglia, G. Venora y G. Muratore, Use of Image Analysis to Evaluate the Shelf Life of Bakery Products, doi: doi.org/10.1016/j.foodres.2014.03.034, Food Research International, 62, 514-522 (2014)

Guiné, R.P., S. Pinho y M.J. Barroca, Study of the Convective Drying of Pumpkin (Cucurbita maxima), doi: 10.1016/j.fbp.2010.09.001, Food and Bioproducts Processing, 89 (4), 422-428 (2011)

Hidalgo, R., M. Gómez, D. Escalera y S. Quisbert, Beneficios de la Guayaba para la Salud Humana, Revista de Investigación e Información en Salud, 10 (25), 27-32 (2015)

Hough, G., I. Wakeling, A. Mucci, E. Chambers, I. Gallardo y L. Alves, Number of Consumers Necessary for Sensory Acceptability Tests, doi: http://dx.doi.org/10.1016/j.foodqual.2005.07.002, Food Quality And Preference,17(6), 522-526 (2006)

International Organization for Standardization, Microbiology of Food and Animal Feeding Stuffs (1999)

Jimenez-Escrig, A., M. Rincón, R. Pullido y F. Saura-Calixto, Guava Fruit (Psidium guajava L.) as a New Source of Antioxidant Dietary Fiber, doi: 10.1021/jf010147p, Journal of Agricultural and Food Chemistry, 49(11), 5489-5493 (2001)

Keen, J.L.H., F.S. Taip, M.N. Ibrahim, N.A. Aziz y M.R.I. Shishir, Effect of Pre-Treatment on the Physical Properties of Pumpkin Powder, Australian Journal of Basic and Applied Sciences, 10 (7), 146-151 (2016)

Martínez, M.M. y J.C. Quintero, Estado Actual de los Desperdicios de Frutas y Verduras en Colombia, 4to Congreso Internacional AmITIC, Popayán, Colombia (2017)

Martins, Z. E., O. Pinho e I.M.P.L.V.O. Ferreira, Food Industry by Products Used as Functional Ingredients of Bakery Products, doi: 10.1016/j.tifs.2017.07.003, Trends in Food Science \& Technology, 67, 106-128 (2017)

Moron, C., I. Zacarias y S.D. Pablo, Producción y Manejo de Datos de Composición Química de Alimentos en Nutrición. In Producción y Manejo de Datos de Composicion Quimica de Alimentos en Nutrición. FAO. Dirección de Alimentación y Nutrición, (1997)

Morris, E.R., N.M. Ptitchkina, L.V. Novokreschonova y G.V. Piskunova, Large Enhancements in Loaf Volume and Organoleptic Acceptability of Wheat Bread by Small Additions of Pumpkin Powder: Possible Role of Acetylated Pectin in Stabilising Gas-Cell Structure, doi: 10.1016/S0268-005X(98)00024-1, Food Hydrocolloids, 12(3), 333-337(1998)

Ndife, J., L.O. Abdulraheem y U.M. Zakari, Evaluation of the Nutritional and Sensory Quality of Functional Breads Produced from Whole Wheat and Soya Bean Flour Blends, African Journal of Food Science, 5(8), 466 - 472 (2011)

O'Shea, N., E.K. Arendt y E. Gallagher, Dietary Fibre and Phytochemical Characteristics of Fruit and Vegetable by Products and their Recent Applications as Novel Ingredients in Food Products, doi: doi.org/10.1016/j.ifset.2012.06.002, Innovative Food Science and Emerging Technologies, 16, 1-10 (2012)

Palacio-Vásquez, E., J.H. Hurtado-lbarbo, J.D. Arroyave-Roa, J., M. Cardona-Caicedo y J. Martínez-Girón, Edulcorantes Naturales Utilizados en la Elaboración de Chocolates, doi: 10.18684/BSAA(15)142-152, Biotecnología en el Sector Agropecuario y Agroindustrial, 15 (2), 142-152 (2017)

Păucean, A. y S. Man, Physico-chemical and Sensory Evaluations of Wheat Bread with Pumpkin (Cucurbita maxima) Pulp Incorporated, J. Agroaliment Proc. Technol., 20(1), 26-32 (2014)

Pongjanta, J., A. Naulbunrang, S. Kawngdang, T. Manon y T. Thepjaikat, Utilization of Pumpkin Powder in Bakery Products. Songklanakarin, J. Sci. Technol, 28(1), 71-79 (2006)

Prabu, S.L., T.N.K. Suriyaprakash, C. Dinesh Kumar, S. Sureshkumar y T. Ragavendran, Nutraceuticals: A Review, www.elixirjournal.org, Elixir Pharmacy, 46, 8372-8377(2012)

Ramírez, M.J., Análisis Sensorial: Pruebas Orientadas al Consumidor, Revisiones de la Ciencia, Tecnología e Ingeniería de los Alimentos, 12 (1), 84-102 (2012)

Ramírez-Navas, J.S. y V. Castro, Análisis de Aceptación y Preferencia del Manjar Blanco del Valle, Biotecnología en el Sector Agropecuario y Agroindustrial, 12(1), 20-27 (2014)

Ribeiro, S.M., J.H. Queiroz, M.E.L.R. de Queiroz, F.M. Campos y H.M.P. Sant'Ana, Antioxidant in Mango (Mangífera indica L.) Pulp, Plant. Foods for Human Nutrition, 62 (1), 13-17 (2007) 
Saeleaw, M., y G. Schleining, Composition, Physicochemical and Morphological Characterization of Pumpkin Flour. In: Proceedings of the ICEF11 $-11^{\text {th }}$ International Congress on Engineering and Food "Food Process Engineering in a Changing World”, ISBN: 978-960-89789-3-5, National Technical University, 869-870 (2011)

Santeramo, F.G., D. Carlucci, B. De Devitiis, A. Seccia, A. Stasi, R. Viscecchia y G. Nardone, Emerging Trends in European Food, Diets and Food Industry, doi: 10.1016/j.foodres.2017.10.039, Food Research International, 1-23 (2017)

See, E.F., W.A. Wan Nadiah y A.A. Noor Aziah, Physico-Chemical and Sensory Evaluation of Breads Supplemented with Pumpkin Flour, ASEAN Food Journal, 14 (2), 123-130 (2007)

Stone, H., y J. L. Sidel, Sensory Evaluation Practices, Elsevier Academic Press, California, USA (2004)

Vera, A.M., y B.C. Hernández, Documento Guía Alimentación Saludable, Santiago de Cali (2013)

Vergara N., E. Granados, E. Agama, J. Tovarb, J. Ruales y L. Bello, Fibre Concentrate from Mango Fruit: Characterization, Associated Antioxidant Capacity and Application as a Bakery Product Ingredient, doi: 10.1016/j.Iwt.2006.02.028, Food Science and Technology, 40 (4), 722-729 (2007)

Zapata, K., F. Cortes y B. Rojano, Polifenoles y Actividad Antioxidante del Fruto de Guayaba Agria (Psidium araca), doi: 10.4067/S0718-07642013000500012, Información Tecnológica, 24 (5), 103 - 112 (2013) 